\title{
Edge condition for long cycles in bipartite graphs
}

\author{
Lech Adamus 1t \\ ${ }^{1}$ Faculty of Applied Mathematics, AGH University of Science and Technology, Al. Mickiewicza 30, 30-059 Kraków, \\ Poland \\ and LRI, Bât. 490, Univeristé Paris-Sud, 91405 Orsay Cedex, France
}

received February 8, 2007, revised January 14, 2009, accepted June 3, 2009.

The following problem was solved by Woodall in 1972: for any pair of nonnegative integers $n$ and $k<\frac{n}{2}-1$ find the minimum integer $g(n, k)$ such that every graph with $n$ vertices and at least $g(n, k)$ edges contains a cycle of length $n-k$. Woodall proved even more: the size $g(n, k)$, in fact, guarantees the existence of cycles $C_{p}$ for all $3 \leq p \leq n-k$.

In the paper an analogous problem for bipartite graphs is considered. It is proved that every bipartite graph with color classes of cardinalities $m$ and $n, m \leq n$, and size greater than $n(m-k-1)+k+1$ contains a cycle of length $2 m-2 k$, where $m \geq \frac{1}{2} k^{2}+\frac{3}{2} k+4, k \in \mathbb{N}$. The bound on the number of edges is best possible. Moreover, this size condition guarantees the existence of cycles of all even lengths up to $2 m-2 k$. We also characterize all extremal graphs for this problem. Finally, we conjecture that the condition on the order may be relaxed to $m \geq 2 k+2$.

Keywords: bipartite graph, cycle, long cycle, bipancyclicity, edge condition, size condition

\section{Introduction}

We will consider only finite, undirected graphs without loops and multiple edges. For the standard terminology and notation see e. g. [4].

Let $G$ be a graph with the vertex set $V(G)$ and the edge set $E(G)$. Denote by $|G|$ the number of vertices in $G$, which is the order of $G$, and by $\|G\|$ the number of edges in $G$, which is the size of $G$.

In 1961, Ore proved the following theorem.

Theorem 1 (Ore [8]) Let $G$ be a graph with $|G|=n$ and $\|G\| \geq \frac{1}{2}(n-1)(n-2)+2$. Then $G$ contains a hamiltonian cycle.

\footnotetext{
${ }^{\dagger}$ The research was partially supported by the AGH University of Science and Technology grant No. 11.420 .04 and by the Polish Ministry of Science doctoral grant No. 0102/M03/2007/32.

1365-8050 (c) 2009 Discrete Mathematics and Theoretical Computer Science (DMTCS), Nancy, France
} 
It is natural to generalize the above problem as follows: for any pair of nonnegative integers $n$ and $k<\frac{n}{2}-1$, find the minimum integer $g(n, k)$, such that every graph of order $n$ and size at least $g(n, k)$ contains a cycle of length $n-k$.

In 1972, Woodall found the number $g(n, k)$ and, what's more, proved that any graph of size at least $g(n, k)$ contains cycles of all lengths up to $n-k$.

Theorem 2 (Woodall [9]) Let $G$ be a graph with $|G|=n \geq 2 k+3, k \in \mathbb{N}$, and

$$
\|G\| \geq g(n, k)=\left(\begin{array}{c}
n-k-1 \\
2
\end{array}\right)+\left(\begin{array}{c}
k+2 \\
2
\end{array}\right)+1 .
$$

Then $G$ contains a cycle of length $p$ for each $p$ such that $3 \leq p \leq n-k$.

This result is best possible. A graph consisting of two cliques, one on $n-k-1$ vertices and the other one on $k+2$ vertices, which share one vertex, is an extremal graph for this problem. It does not contain any cycle of length $n-k$ and it has exactly $g(n, k)-1$ edges.

We wish to investigate an analogous problem for bipartite graphs.

Let $G=(X, Y ; E)$ denote a bipartite simple graph with color classes $X$ and $Y$ and the set of edges $E$. Such a graph $G$ is called balanced if $X$ and $Y$ have equal cardinalities.

For $x \in V(G)$ we denote by $N_{G}(x)$ the set of neighbors of the vertex $x$ in $G$, and by $d_{G}(x)$ the degree of the vertex $x$ in $G$, so that $d_{G}(x)=\left|N_{G}(x)\right|$. The minimal vertex degree in $G$ will be denoted by $\delta(G)$. For $A \subset V(G)$ and $x \in V(G)$ we define $e(x, A)=\left|N_{G}(x) \cap A\right|$. We write $G-A$ for the subgraph of $G$ induced by $V(G) \backslash A$.

An $x y$ - path is a path with endvertices $x$ and $y$, and $C_{k}$ (resp. $P_{k}$ ) denotes a cycle (resp. path) of order $k$. The balanced bipartite graph $G=(X, Y ; E)$ is hamiltonian biconnected if for any two vertices $x \in X$ and $y \in Y$, there exists a hamiltonian $x y$-path in $G$. It is 2-hamiltonian biconnected if for any pair of vertices $a \in X$ and $b \in Y$, the subgraph $G-\{a, b\}$ is hamiltonian biconnected. A graph $G$ is said to be bipancyclic if it contains cycles of all even lengths up to $|G|$.

The aim of this paper is to find the minimum number of edges of a bipartite graph $G=(X, Y ; E)$ on $m+n$ vertices $(|X|=m \leq n=|Y|)$, which guarantees the existence of a cycle of length $2 m-2 k$ in $G$, for any integer $0 \leq k<\frac{m}{2}-1$. We prove that, for $m \geq \frac{1}{2} k^{2}+\frac{3}{2} k+4$, this extremal number of edges is equal to $n(m-k-1)+k+2$ and this size, in fact, guarantees the existence of cycles of all even lengths up to $2 m-2 k$ in $G$. Our result is a generalization of the following theorems.

Theorem 3 (Mitchem and Schmeichel [7]) Let $G=(X, Y ; E)$ be a balanced bipartite graph of order $2 n(|X|=|Y|=n)$. If $\| G|| \geq n^{2}-n+2$, then $G$ is bipancyclic.

Theorem 4 (Bagga and Varma [3]) Let $G=(X, Y ; E)$ be a bipartite graph with $|X|=m \leq n=|Y|$. If $\|G\| \geq n(m-1)+2$, then $G$ contains cycles $C_{2 p}$ for all $2 \leq p \leq m$.

We also characterize all extremal graphs of size $n(m-k-1)+k+1$ that do not contain any cycle of length $2 m-2 k$.

The main result of this paper (Theorem 5) and its proof are presented in Section 2. A version for balanced bipartite graphs of this theorem was first announced in [1]. In Section 3 we formulate a conjecture which is a stronger form of Theorem 5 


\section{Main theorem}

First we introduce a family of extremal graphs. For any three integers $m, n$ and $k$, where $2 \leq m \leq n$ and $0 \leq k \leq m-2$, we define the family $\mathcal{G}_{m, n, k}$ of bipartite graphs $G=(X, Y ; E)$ with $|X|=m,|Y|=n$, such that $X=A \cup B,|B|=k+1, d_{G}(v)=n$ for every $v \in A$ and $d_{G}(w)=1$ for every $w \in B$ (see Figure 1). Note that every graph of the family $\mathcal{G}_{m, n, k}$ has size equal to $n(m-k-1)+k+1$ and does not contain any cycle of length $2 m-2 k$. Moreover, if we add any edge to a graph of $\mathcal{G}_{m, n, k}$, then it will contain a cycle $C_{2 m-2 k}$.

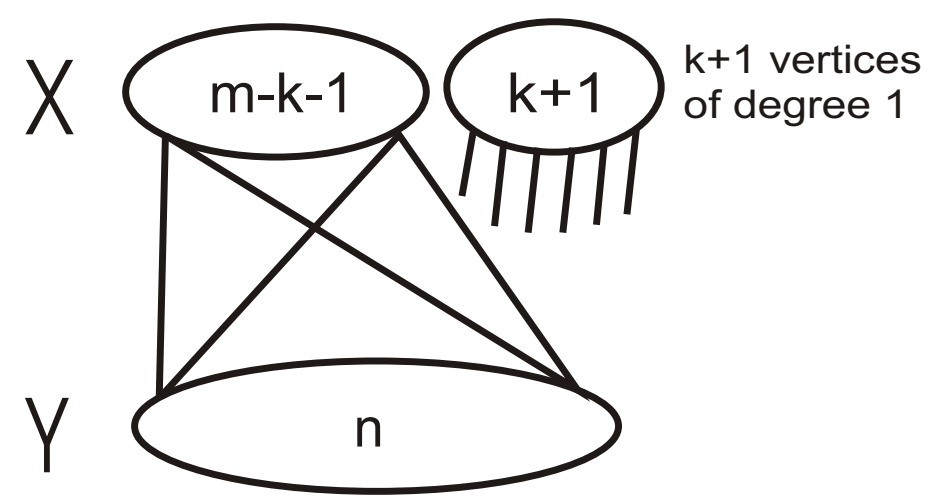

Fig. 1: The family $\mathcal{G}_{m, n, k}$ of extremal graphs.

Our purpose is to prove the following theorem.

Theorem 5 Let $G=(X, Y ; E)$ be a bipartite graph with $|X|=m \leq n=|Y|$, where $m \geq \frac{1}{2} k^{2}+\frac{3}{2} k+4$, $k \in \mathbb{N}$. If

$$
\|G\| \geq f(m, n, k)=n(m-k-1)+k+1,
$$

then either $G$ contains a cycle of length $2 m-2 k$, or else $\|G\|=f(m, n, k)$ and $G$ is isomorphic to a graph of the family $\mathcal{G}_{m, n, k}$.

In both cases $G$ contains $C_{2 p}$ for all $2 \leq p \leq m-k-1$.

In the course of the proof of Theorem 5 , we shall use the following results.

Theorem 6 (Bagga and Varma [3]) Let $G=(X, Y ; E)$ be a balanced bipartite graph of order $2 n$. If for every pair of nonadjacent vertices $x \in X$ and $y \in Y, d_{G}(x)+d_{G}(y) \geq n+1$, then $G$ is bipancyclic.

Theorem 7 (Amar, Favaron, Mago and Ordaz [2]) Let $G=(X, Y ; E)$ be a balanced bipartite graph of order $2 n$. If $\|G\| \geq n^{2}-n+3$, then $G$ is hamiltonian biconnected and 2-hamiltonian biconnected. 
Proof: The proof will be divided into two steps. We first show that Theorem 5 is true for $m=n$. In the second step we will consider the situation when $m<n$.

Step 1. $m=n$

We will proceed by induction on $k$.

We assume, without loss of generality, that the size of $G$ is equal to $f(n, n, k)$, for otherwise we may consider a spanning subgraph of $G$ with exactly $f(n, n, k)$ edges instead of $G$.

Let $k=0$. We want to show that if $G$ is a balanced bipartite graph with $|G|=2 n \geq 8$ and $\|G\|=$ $n^{2}-n+1$ then $G$ is bipancyclic unless $G \in \mathcal{G}_{n, n, 0}$.

Applying Theorem 6 to the graph $G$, we get that either $G$ is bipancyclic, or else there are two nonadjacent vertices $x \in X$ and $y \in Y$ such that $d_{G}(x)+d_{G}(y) \leq n$. But then $\|G-\{x, y\}\| \geq n^{2}-n+1-n=$ $(n-1)^{2}$, so $G-\{x, y\}$ is a complete balanced bipartite graph on $2 n-2$ vertices (hence $G$ contains cycles of all even lengths up to $2 n-2$ ). If so, then, in fact, $d_{G}(x)+d_{G}(y)=n$. Since $x$ and $y$ are not adjacent, their degrees are both greater than zero. In case $d_{G}(x) \geq 2$ and $d_{G}(y) \geq 2$ it is easy to find a hamiltonian cycle in $G$. It remains to consider the case when one of the vertices $x$ and $y$, say $x$, has degree 1 . Then $y$ has degree $n-1$ and is adjacent to every vertex of the set $X \backslash\{x\}$, which implies $G \in \mathcal{G}_{n, n, 0}$.

Now assume the theorem holds for $k-1, k \geq 1$; we will prove that it holds also for $k$.

Let us start with the observation that $f(n, n, k)-f(n-1, n-1, k-1)=n-k$. In the proof we shall consider two cases.

Case 1. Suppose there are two vertices $x \in X$ and $y \in Y$ in the graph $G$ such that $d_{G}(x)+d_{G}(y) \leq n-k$.

Then $G-\{x, y\}$ is a balanced bipartite graph with $2 n-2$ vertices and at least $f(n, n, k)-$ $(n-k)=f(n-1, n-1, k-1)$ edges. By the inductive hypothesis there are cycles of all even lengths up to $2(n-1)-2(k-1)=2 n-2 k$ contained in $G-\{x, y\}$, hence also in $G$, unless $G-\{x, y\} \in \mathcal{G}_{n-1, n-1, k-1}$. In the latter case $d_{G}(x)+d_{G}(y)=n-k$ and $x$ and $y$ are not adjacent, because $\|G-\{x, y\}\|=f(n-1, n-1, k-1)$. Moreover, $X \backslash\{x\}=A \cup B$, where $A=\left\{v: d_{G-\{x, y\}}(v)=n-1\right\}, B=\left\{v: d_{G-\{x, y\}}(v)=1\right\}$ and $|B|=k$. We see at once that cycles of all even lengths up to $2 n-2 k-2$ are contained in $G-\{x, y\}$, hence also in $G$. What remains to show is that either $C_{2 n-2 k} \subset G$ or $G \in \mathcal{G}_{n, n, k}$.

It is easy to check that $C_{2 n-2 k} \subset G$ in case $d_{G}(x) \geq 2$. It remains to consider what happens when $d_{G}(x) \leq 1$. Suppose that $d_{G}(x)=0$. Then $d_{G}(y)=n-k>k$, which implies that there is at least one vertex in the set $A$, say $v_{1}$, and at least one vertex, say $v_{2}$, in the set $B$ which are adjacent to the vertex $y$. In this case it is a simple matter to find a cycle $C_{2 n-2 k}$ contained in $G$ which passes through the vertices $v_{1}, y$ and $v_{2}$. So suppose that $d_{G}(x)=1$, which implies $d_{G}(y)=n-k-1>k$. Then either the vertex $y$ is adjacent to all vertices in the set $A$ (which means that $G \in \mathcal{G}_{n, n, k}$ ), or, again, $y$ is adjacent to some vertex of $A$ and to at least one vertex in $B$, and we again find a cycle of length $2 n-2 k$ contained in $G$.

Case 2. Suppose for every pair of vertices $x \in X$ and $y \in Y$ in the graph $G$, we have $d_{G}(x)+d_{G}(y) \geq$ $n-k+1$.

Firstly, observe that this assumption implies that $\delta(G) \geq 2$, for otherwise $G$ has at least $n(n-k)$ edges, which contradicts the assumption made at the beginning of Step 1 that $\|G\|=f(n, n, k)$. 
Suppose now that there exists $2 \leq p \leq n-k$ such that $G$ does not contain any cycle of length $2 p$. Theorem 6 implies that there are two nonadjacent vertices $x_{1} \in X$ and $y_{1} \in Y$ such that $d_{G}\left(x_{1}\right)+$ $d_{G}\left(y_{1}\right) \leq n$. Let $G_{1}:=G-\left\{x_{1}, y_{1}\right\}$. Observe that $\left\|G_{1}\right\| \geq f(n, n, k)-n$. Now, we apply Theorem 6 to the graph $G_{1}$. Since there exists some $p \in\{2, \ldots, n-k\}$ such that $C_{2 p} \not \subset G$, the subgraph $G_{1}$ of $G$ does not contain $C_{2 p}$ and, in particular, $G_{1}$ is not bipancyclic. Thus, there are two nonadjacent vertices $x_{2} \in X \cap V\left(G_{1}\right)$ and $y_{2} \in Y \cap V\left(G_{1}\right)$ such that $d_{G_{1}}\left(x_{2}\right)+d_{G_{1}}\left(y_{2}\right) \leq n-1$. Write $G_{2}:=G-\left\{x_{1}, y_{1}, x_{2}, y_{2}\right\}$. We have $\left\|G_{2}\right\| \geq\left\|G_{1}\right\|-(n-1) \geq f(n, n, k)-n-(n-1)$. We now apply Theorem 6 to the graph $G_{2}$, and so on. After $k+1$ steps we finally get the graph $G_{k+1}:=G-\left\{x_{1}, x_{2}, \ldots, x_{k+1}, y_{1}, y_{2}, \ldots, y_{k+1}\right\}$ such that $\left\|G_{k+1}\right\| \geq\left\|G_{k}\right\|-(n-k) \geq$ $f(n, n, k)-n-(n-1)-\ldots-(n-k)=f(n, n, k)-\frac{2 n-k}{2} \cdot(k+1)=n^{2}-2 n k-2 n+\frac{1}{2} k^{2}+$ $\frac{3}{2} k+1$. For $n \geq \frac{1}{2} k^{2}+\frac{3}{2} k+4$, we have

$$
n^{2}-2 n k-2 n+\frac{1}{2} k^{2}+\frac{3}{2} k+1 \geq(n-k-1)^{2}-(n-k-1)+3,
$$

and $G_{k+1}$ is hamiltonian biconnected and 2-hamiltonian biconnected by Theorem 7 , and also bipancyclic by Theorem 3. It follows that there are cycles of all even lengths up to $2 n-2 k-2$ contained in $G_{k+1}$, hence also in $G$. So the hypothesis, that there exists $2 \leq p \leq n-k$ such that $C_{2 p} \not \subset G$, implies that $G$ does not contain any cycle of length $2 n-2 k$.

Let $V^{\prime}:=V(G) \backslash V\left(G_{k+1}\right)$. We need to consider several subcases.

Subcase 2.1. Assume there are two vertices $x \in X \cap V^{\prime}$ and $y \in Y \cap V^{\prime}$ such that $e\left(x, V^{\prime}\right)=e\left(y, V^{\prime}\right)=0$.

Since $d_{G}(x)+d_{G}(y) \geq n-k+1$, there is a vertex $u \in Y \cap V\left(G_{k+1}\right)$ which is adjacent to $x$, and there is a vertex $v \in X \cap V\left(G_{k+1}\right)$ which is adjacent to $y$. There exists a $u v$-path of order $2 n-2 k-2$ in $G_{k+1}$, because $G_{k+1}$ is hamiltonian biconnected. It means that there is a path $P_{2 n-2 k}=x^{1} y^{1} x^{2} y^{2} \ldots x^{2 n-2 k} y^{2 n-2 k}\left(x^{1}=x, y^{1}=u, x^{2 n-2 k}=v, y^{2 n-2 k}=y\right)$ contained in $G$, which passes through all vertices of the graph $G_{k+1}$. Since $C_{2 n-2 k} \not \subset G$, if $x^{1} y^{i} \in E$ then $x^{i} y^{2 n-2 k} \notin E$, for otherwise $x^{1} y^{1} x^{2} y^{2} \ldots x^{i} y^{2 n-2 k} x^{2 n-2 k} \ldots y^{i} x^{1}$ is a cycle of length $2 n-2 k$ contained in $G$. Thus, we have $e\left(x, V\left(G_{k+1}\right)\right)+e\left(y, V\left(G_{k+1}\right)\right) \leq n-k$, which, in this case, means that $d_{G}(x)+d_{G}(y) \leq n-k$, contradicting the assumption.

Subcase 2.2. Assume for every pair of vertices $x \in X \cap V^{\prime}$ and $y \in Y \cap V^{\prime}$, we have $e\left(x, V^{\prime}\right) \neq 0$ or $e\left(y, V^{\prime}\right) \neq 0$.

We then consider an edge $x y$ with $x \in X \cap V^{\prime}, y \in Y \cap V^{\prime}$.

Subcase 2.2.1. Suppose for at least one edge $x y$ with $x \in X \cap V^{\prime}, y \in Y \cap V^{\prime}$, we have $e\left(x, V\left(G_{k+1}\right)\right) \geq 1$ and $e\left(y, V\left(G_{k+1}\right)\right) \geq 1$.

Then, again, there is a vertex $u \in Y \cap V\left(G_{k+1}\right)$ which is adjacent to $x$, and there is a vertex $v \in X \cap V\left(G_{k+1}\right)$ which is adjacent to $y$, and there exists a $u v$-path of order $2 n-2 k-2$ in $G_{k+1}$. We can extend this path to a cycle of length $2 n-2 k$ using edges $u x, x y, y v$, a contradiction with the assumption that $C_{2 n-2 k} \not \subset G$.

Subcase 2.2.2. Suppose for every edge $x y$ with $x \in X \cap V^{\prime}, y \in Y \cap V^{\prime}$, we have $e\left(x, V\left(G_{k+1}\right)\right)=0$ or $e\left(y, V\left(G_{k+1}\right)\right)=0$.

Without loss of generality we may assume that $e\left(x, V\left(G_{k+1}\right)\right)=0$.

We show that $e\left(u, V\left(G_{k+1}\right)\right) \geq 2$ for every vertex $u \in Y \cap V^{\prime}$. We have $d_{G}(x) \leq k$ (because 
$x$ is joined only with at most $k$ vertices of $V^{\prime}$, which is a consequence of the construction of $\left.G_{k+1}\right)$, so, by the assumption made at the beginning of Case $2, d_{G}(u) \geq n-2 k+1$ for every vertex $u \in Y \cap V^{\prime}$. For $n \geq \frac{1}{2} k^{2}+\frac{3}{2} k+4$, it follows that $d_{G}(u) \geq k+2$, which leads to $e\left(u, V\left(G_{k+1}\right)\right) \geq 2$, since $u \in Y \cap V^{\prime}$ has at least one non-neighbour in $X \cap V^{\prime}$.

Since $\delta(G) \geq 2$, the vertex $x$ has at least two neighbors in $V^{\prime}$, say $y, y^{\prime}$. By the above observation, there are two different vertices $v_{1}, v_{2} \in X \cap V\left(G_{k+1}\right)$ such that $v_{1} y \in E$ and $v_{2} y^{\prime} \in E$. $G_{k+1}$ is hamiltonian, so there exists a vertex $u \in Y \cap V\left(G_{k+1}\right)$ such that $v_{1} u \in E$. Let $a \in Y \cap V\left(G_{k+1}\right)$ and $a \neq u$. Since $G_{k+1}$ is 2-hamiltonian biconnected, $G_{k+1}-\left\{v_{1}, a\right\}$ is hamiltonian biconnected, so there exists a $v_{2} u$-path of order $2 n-2 k-4$ in $G_{k+1}-\left\{v_{1}, a\right\}$. We can extend this path to a cycle of length $2 n-2 k$ using edges $v_{2} y^{\prime}, y^{\prime} x, x y, y v_{1}, v_{1} u$, a contradiction with the assumption that $C_{2 n-2 k} \not \subset G$.

Step 2. $m<n$

First order the vertices of $Y=\left\{y^{1}, \ldots, y^{n}\right\}$ so that $d_{G}\left(y^{1}\right) \leq \ldots \leq d_{G}\left(y^{n}\right)$. Put $Y^{\prime}:=\left\{y^{1}, \ldots, y^{n-m}\right\}$. We shall consider two cases depending on the degree of the vertex $y^{n-m}$.

Case 1. $d_{G}\left(y^{n-m}\right) \geq m-k$

Then $G-Y^{\prime}$ is a balanced bipartite graph on $2 m$ vertices such that $\left\|G-Y^{\prime}\right\| \geq m(m-k)>$ $f(m, m, k)$ since $m>k+1$. From what has already been proved in Step 1, we conclude that $G-Y^{\prime} \supset C_{2 p}$ for all $2 \leq p \leq m-k$, which completes the proof in this case.

Case 2. $d_{G}\left(y^{n-m}\right) \leq m-k-1$

Now we have

$$
\left\|G-Y^{\prime}\right\| \geq f(m, n, k)-(n-m)(m-k-1)=f(m, m, k)
$$

which again implies $G-Y^{\prime} \supset C_{2 p}$ for all $2 \leq p \leq m-k-1$, and also $G-Y^{\prime} \supset C_{2 m-2 k}$ unless $\left\|G-Y^{\prime}\right\|=f(m, m, k)$ and $G-Y^{\prime} \in \mathcal{G}_{m, m, k}$. Therefore it remains to consider the situation when $X=A \cup B$, where $A=\left\{v: d_{G-Y^{\prime}}(v)=m\right\}, B=\left\{v: d_{G-Y^{\prime}}(v)=1\right\},|B|=k+1$. Then every vertex of $Y^{\prime}$ has degree in $G$ equal $m-k-1$ since inequality 1 is, in fact, an equality.

Suppose now that no cycle of length $2 m-2 k$ is contained in $G$. Consequently, no vertex of $Y^{\prime}$ can be adjacent to both a vertex of $A$ and a vertex of $B$, for otherwise we could simply construct a cycle $C_{2 m-2 k}$ in $G$. Hence the assumption: $d_{G}\left(y^{i}\right)=m-k-1$ for all $1 \leq i \leq n-m$, implies (for $m>2 k+2$ ) that every vertex of $Y^{\prime}$ is adjacent to all vertices of $A$. It follows that $G \in \mathcal{G}_{m, n, k}$, which completes the proof.

\section{Conjecture}

We expect that the condition on the order of $G$ in Theorem 5 may be relaxed. We formulate the following conjecture.

Conjecture 1 Let $G=(X, Y ; E)$ be a bipartite graph with $|X|=m \leq n=|Y|$, where $m \geq 2 k+2$, $k \in \mathbb{N}$. If $\|G\|>n(m-k-1)+k+1$, then $G$ contains cycles of all even lengths up to $2 m-2 k$. 
Notice that the assumption $m \geq 2 k+2$ of the conjecture is weakest possible, as shown by the following example.

Example 1 Let $G_{1}=(X, Y ; E)$ be a bipartite graph with vertex classes of the form $X=Q \cup R$, $Y=S \cup T$, where $|Q|=|T|=m-k-1,|R|=k+1,|S|=n-m+k+1, m \leq n$. Fix a vertex $y_{0}$ in $T$, and let $N_{G_{1}}(v)=S \cup\left\{y_{0}\right\}$ for all $v \in Q$, and $N_{G_{1}}(w)=T$ for all $w \in R$. Then $\left\|G_{1}\right\|>n(m-k-1)+k+1$ for $k+3 \leq m \leq 2 k+1$, yet $G_{1}$ contains no cycle of length greater than $2 m-2 k-2$. Hence the necessity of the assumption $m \geq 2 k+2$.

There are some results which suggest that this conjecture may be true.

It is true for $k=0$ (Theorem 4). We will show that it is true also for $k=1$ but we will need the following result (stronger than Theorem 3).

Theorem 8 Let $G=(X, Y ; E)$ be a balanced bipartite graph of order $2 n$. If $\|G\| \geq n^{2}-n+1$, then $G$ is bipancyclic or $G \in \mathcal{G}_{n, n, 0}$.

The proof of Theorem 8 is the same as that of Theorem 5 in the case $k=0$ in Step 1 .

Now we are ready to prove Conjecture 1 for $k=1$.

Sketch of proof of Conjecture 1; An analysis similar to that in Step 2 of the proof of Theorem 5 shows that Conjecture 1 is true in general case $(m \leq n)$ if it is true for balanced bipartite graphs $(m=n)$. Therefore it remains only to consider the situation when $m=n$.

Suppose that there exists $2 \leq p \leq n-1$ such that $G$ does not contain any cycle of length $2 p$. Then, by Theorem 6 there are two nonadjacent vertices $x \in X$ and $y \in Y$ such that $d_{G}(x)+d_{G}(y) \leq n$. Hence $\|G-\{x, y\}\| \geq n^{2}-2 n+3-n=(n-1)^{2}-(n-1)+1$. By Theorem $8, G-\{x, y\} \in \mathcal{G}_{n-1, n-1,0}$, and therefore contains cycles of all even lengths up to $2 n-4$. Since $\|G-\{x, y\}\|=n^{2}-3 n+3$, we must have $d_{G}(x)+d_{G}(y)=n$. But the assumption $C_{2 n-2} \not \subset G$ implies that $d_{G}(x) \leq 1$ and $d_{G}(y) \leq n-2$, a contradiction.

In 1985, Jackson [6] found the number of edges of a bipartite graph $G$ needed to ensure the existence of a cycle of length at least $2 p$ in $G$. Conjecture 1, if true, would be a significant strengthening of the following theorem.

Theorem 9 (Jackson [6]) Let $G=(X, Y ; E)$ be a bipartite graph with $|X|=m \leq n=|Y|$, where $m \geq 2 k+2, k \in \mathbb{N}$. If $\|G\| \geq n(m-k-1)+k+2$, then there exists $p \geq m-k$ such that $C_{2 p} \subset G$.

There is another result which suggest that Conjecture 1 may be true. Gyárfás, Rousseau and Schelp solved an analogous problem for paths in bipartite graphs. They characterized all extremal graphs for this problem, which are similar to those of the family $\mathcal{G}_{m, n, k}$.

Theorem 10 (Gyárfás, Rousseau and Schelp [5]) Let $G=(X, Y ; E)$ be a bipartite graph with $|X|=$ $m \leq n=|Y|$, where $m>2 k+2, k \in \mathbb{N}$. If $\|G\| \geq n(m-k-1)$, then either $P_{2 m-2 k} \subset G$ or $\|G\|=n(m-k-1)$ and $G$ is the exceptional graph for which $X=A \cup B, A=\left\{x \in X: d_{G}(x)=n\right\}$, $B=\left\{x \in X: d_{G}(x)=0\right\},|B|=k+1$. 


\section{Acknowledgements}

The author wishes to express his thanks to Irmina Zioło, Paweł Wojda and Jakub Przybyło for their helpful and valuable remarks.

\section{References}

[1] L. Adamus, Long cycles in graphs and digraphs, Electron. Notes Discrete Math. 24 (2006) 3-7, www.elsevier.com/locate/endm.

[2] D. Amar, O. Favaron, P. Mago and O. Ordaz, Biclosure and bistability in a balanced bipartite graph, J. Graph Theory 20 (1995) 513-529.

[3] K.S. Bagga and B.N. Varma, Hamiltonian properties in bipartite graphs, Bull. Inst. Comb. Appl. 26 (1999) 71-85.

[4] R. Diestel, Graph Theory, Springer-Verlag, New York, 1997.

[5] A. Gyárfás, C. C. Rousseau and R. H. Schelp, An extremal problem for paths in bipartite graphs, J. Graph Theory 8 (1984) 83-95.

[6] B. Jackson, Long cycles in bipartite graphs, J. Comb. Theory Ser. B 38 (1985) 118-131.

[7] J. Mitchem and E. Schmeichel, Pancyclic and bipancyclic graphs - a survey, Graphs and applications, Proc. 1st Symp. Graph theory, Boulder/Colo. (USA) 1982 (ed. F. Harary and J.S. Maybee), Wiley, New York, 1985, 271-278.

[8] O. Ore, Arc coverings of graphs, Ann. Mat. Pura Appl. 55 (1961) 315-321.

[9] D.R. Woodall, Sufficient conditions for circuits in graphs, Proc. London Math. Soc. 24 (1972) 739-755. 